\title{
Pharmacovigilance for Novel Oral Anticoagulants: Why is It So Crucial?
}

\section{Eugenia Ch Yiannakopoulou*}

Department of Medical Laboratories Faculty of Health and Caring Professions Technological Educational Institute of Athens, Athens, Greece

Novel oral anticoagulants (NOACs) inhibit key enzymes in the coagulation cascade namely thrombin and activated factor X (FXa). NOACs already approved are the thrombin inhibitor dabigatran etexilate and the FXa inhibitors rivaroxaban and apixaban. These drugs have been licensed for the treatment of deep vein thrombosis and for the treatment of non valvular atrial fibrillation. Non-valvular atrial fibrillation is a quite common arrhythmia and its prevalence increases with age. According to epidemiological data, non valvular atrial fibrillation is more common in people older than 80 years old. People suffering from atrial fibrillation have increased risk of embolic stroke. Chronic treatment with oral anticoagulants reduces the risk of stroke. Oral anticoagulation therapy is recommended for patients with two or more risk factors for thromboembolism in addition to atrial fibrillation. Patients with atrial fibrillation and a single additional risk factor should be evaluated on an individual basis for the possible need of anticoagulant treatment. Anticoagulant treatment is not recommended for people with atrial fibrillation and no other risk factors. Until recently, patients with atrial fibrillation were treated with warfarin or closely related vitamin $\mathrm{K}$ antagonists. However, these medicines need tight coagulation monitoring as their pharmacokinetics cannot be predicted.

In contrast with vitamin $\mathrm{K}$ antagonists, NOACs have rapid onset of action, short half-life in plasma, limited drug - drug interactions and no need of routine coagulation monitoring for dose adjustment, because their anticoagulant effect is sufficiently constant at fixed oral doses. Based on data from phase III randomized controlled trials comparing NOACs with warfarin, NOACs are at least as effective as warfarin for preventing ischemic stroke in patients with non-valvular atrial fibrillation; they carry a lower risk of cerebral haemorrhage, especially intracranial haemorrhage and are more practical in use. Dose adjustment is needed for patients suffering from chronic renal insufficiency. Until now, there are no head to head comparisons between any two NOACs.

It is well known, that, when a novel drug enters the market, the safety of the drug is not well established. NOACs are already prescribed to old patients with comorbidities who are often treated with multiple drugs. These people are often frail are not really represented in randomized controlled clinical trials. Thus, pharmacovigilance is crucial for the monitoring of the safety of these novel medicines. Gastrointestinal hemorrhage is the most common adverse event of these drugs. However non gastrointestinal haemorrhage could be anticipated. Non haemorrhagic adverse events are also possible. Liver failure has been reported as an adverse event associated with the use of NOACs. Yet, given that NOACs are prescribed for prophylaxis and not for treatment, safety concerns always exist.

Physicians should be familiar with the pharmacology of NOACs, dose adjustments, contra-indications, drug-drug interactions. They should follow patients treated with NOACs and they should report any possible adverse event to appropriate pharmacovigilance agents. Patients should also be well informed for the possible adverse events. All the medical community and the patients should contribute to the demonstration of the safety of the NOACs through their active involvement to pharmacovigilance efforts.
${ }^{*}$ Corresponding author: Eugenia Yiannakopoulou, Eleutheriou Benizelou 106 Kallithea 17676, Athens Greece, Tel: 0030 2109563791; 0030 210-9563761; E-mail: nyiannak@teiath.gr

Received March 30, 2015; Accepted March 31, 2015; Published April 06, 2015

Citation: Yiannakopoulou ECh (2015) Pharmacovigilance for Novel Ora Anticoagulants: Why is It So Crucial?. J Pharmacovigilance 3: e135. doi:10.4172/2329$6887.1000 \mathrm{e} 135$

Copyright: ( 2015 Yiannakopoulou ECh. This is an open-access article distributed under the terms of the Creative Commons Attribution License, which permits unrestricted use, distribution, and reproduction in any medium, provided the original author and source are credited. 